\title{
A NON-PARAMETRIC METHOD TO TEST EQUALITY OF INTERMEDIATE LATENT ROOTS OF TWO POPULA- TIONS IN A PRINCIPAL COMPONENT ANALYSIS
}

\author{
Takakazu Sugiyama* and Kenji Ushizawa**
}

\begin{abstract}
We propose a nonparametric criterion to test the hypothesis that the $j$-th largest roots of two populations are equal, in Section 2. This testing procedure is distribution free, and in Section 3 and 4 we show that it is reliable when the sample sizes increase and the population latent roots are separate, under the multivariate normal distribution. We suggest which sample sizes are necessary to rely on the above test.
\end{abstract}

Key Words and Phrases: nonparametric method, principal component analysis, equality of intermediate latent roots, Ansari-Bradley test, multivariate normal distribution.

\section{Introduction}

Principal component analysis (PCA) is a useful and important multivariate technique not only from a practical but also from a theoretical point of view. Its general objectives are data reduction and interpretation. The mathematical treatment of PCA is based on latent roots and vectors. Although many books on PCA have been published ([1], [4], [5]), because of the difficulties to obtain the exact distribution, we have not seen the two-sample problem to test the hypothesis that the $j$-th largest roots are equal. In this paper, we propose a new testing procedure for the above test using principal component scores.

Suppose that $\boldsymbol{x}_{1}^{(1)}, \boldsymbol{x}_{2}^{(1)}, \cdots, \boldsymbol{x}_{N_{1}}^{(1)}$ and $\boldsymbol{x}_{1}^{(2)}, \boldsymbol{x}_{2}^{(2)}, \cdots, \boldsymbol{x}_{N_{2}}^{(2)}$ are random observations from $p$-variate distributions $\Lambda_{p}\left(\boldsymbol{\mu}_{1}, \Sigma_{1}\right)$ with mean $\boldsymbol{\mu}_{1}$ and covariance matrix $\boldsymbol{\Sigma}_{1}$ and $\Lambda_{p}\left(\mu_{2}, \Sigma_{2}\right)$ with mean $\mu_{2}$ and covariance matrix $\Sigma_{2}$ respectively. Let $\lambda_{j}^{(1)}$ and $\lambda_{j}^{(2)}$ be the $j$-th largest latent roots in the first and the second population, respectively. For a fixed $j$, we consider the hypothesis testing problem

$$
H_{0}: \lambda_{j}^{(1)}=\lambda_{j}^{(2)} \text { against } H_{1}: \lambda_{j}^{(1)} \neq \lambda_{j}^{(2)} .
$$

One of the testing criteria may be the $j$-th largest sample latent roots $l_{j}^{(1)}$ and $l_{j}^{(2)}$ of each sample covariance matrix given by

$$
\boldsymbol{S}^{(k)}=\frac{1}{N_{k}-1} \sum_{m=1}^{N_{k}}\left(\boldsymbol{x}_{m}^{(k)}-\overline{\boldsymbol{x}}^{(k)}\right)\left(\boldsymbol{x}_{m}^{(k)}-\overline{\boldsymbol{x}}^{(k)}\right)^{\prime}, k=1,2,
$$

where $\overline{\boldsymbol{x}}^{(k)}$ is the sample mean vector. If the difference between $l_{j}^{(1)}$ and $l_{j}^{(2)}$ is large, we may reject the null hypothesis $H_{0}$, otherwise we may not reject it. To

Received September, 1997. Revised November, 1997, March, 1998, May, 1998. Accepted June, 1998.

- Department of Mathematics, Faculty of Science and Engineering, Chuo University, 1-1327 Kasuga, Bunkyoku, Tokyo 112-8551, Japan.

** Department of Management and Informatics, Sanno College, 1573 Kamikasuya, Isehara, Kanagawa 259-1197, Japan. 
obtain the critical region, we need the joint distribution of $l_{j}^{(1)}$ and $l_{j}^{(2)}$. Except to the case of the largest latent roots $l_{1}^{(1)}$ and $l_{2}^{(2)}$ it is difficult to obtain the exact joint distribution under the multivariate normal distribution. In the principal component analysis there are many parameters to be considered. When we do not have enough information about the population parameters, we may be interested in the nonparametric test.

Let $\boldsymbol{h}_{j}^{(1)}$ and $\boldsymbol{h}_{j}^{(2)}$ be the sample latent vectors corresponding to the $j$-th largest latent roots $l_{j}^{(1)}$ and $l_{j}^{(2)}$ respectively. As $l_{j}^{(1)}$ and $l_{j}^{(2)}$ are the variances of principal components

$$
y_{j a}^{(1)}=\boldsymbol{h}_{j}^{(1)} \boldsymbol{x}_{\alpha}^{(1)}, \quad \alpha=1,2, \cdots, N_{1},
$$

and

$$
y_{j \alpha}^{(2)}=\boldsymbol{h}_{j}^{(2)} \boldsymbol{x}_{\alpha}^{(2)}, \quad \alpha=1,2, \cdots, N_{2},
$$

respectively, we consider the application of a nonparametric test for equal variance in next Section.

\section{Testing procedure}

Our problem is to test the hypothesis that the $j$-th largest latent roots in the two groups are equal, using the principal component scores adjusted locations by the means

$$
Y_{1}=\left\{y_{j 1}^{(1)}-\bar{y}_{j}^{(1)}, y_{j 2}^{(1)}-\bar{y}_{j}^{(1)}, y_{j 3}^{(1)}-\bar{y}_{j}^{(1)}, \cdots, y_{j N_{1}}^{(1)}-\bar{y}_{j}^{(1)}\right\}
$$

and

$$
Y_{2}=\left\{y_{j 1}^{(2)}-\bar{y}_{j}^{(2)}, y_{j 2}^{(2)}-\bar{y}_{j}^{(2)}, y_{j 3}^{(2)}-\bar{y}_{j}^{(2)}, \cdots, y_{j N_{2}}^{(2)}-\bar{y}_{j}^{(2)}\right\} .
$$

When the populations are distributed as multivariate normal, we apply the Ansari-Bradley $(\mathrm{A}-\mathrm{B})$ test to the above data as following procedure:

(1) Two samples $Y_{1}$ and $Y_{2}$ are combined and ordered, and then ranks are assigned as follows:

$$
\left\{1,2, \cdots,\left(N_{1}+N_{2}\right) / 2,\left(N_{1}+N_{2}\right) / 2, \cdots, 2,1\right\}
$$

if $N_{1}+N_{2}$ is even and

$$
\left\{1,2, \cdots,\left(N_{1}+N_{2}-1\right) / 2,\left(N_{1}+N_{2}+1\right) / 2,\left(N_{1}+N_{2}-1\right) / 2, \cdots, 2,1\right\}
$$

if $N_{1}+N_{2}$ is odd.

(2) Calculate the sum of the above ranks, $W$, associated with the sample $Y_{1}$. The following statistic based $W$

$$
Z=\frac{W-E(W)}{\sqrt{V(W)}}
$$

can be approximated by a standard normal distribution $N(0,1)$ according to Ansari-Bradley (1960) under a few assumptions. One of the assumptions for the $A-B$ test is that the sample values are pairwise independent. But, when we use the sample latent vector $\boldsymbol{h}_{j}^{(k)}$ to calculate the $y_{j i}^{(k)}$, they are no longer 
independent, though the degree of dependence is very weak. What kind of nonparametric test is available depends on the population situation. As we will see later, the A-B test is useful for the multivariate normal distribution.

Now we will evaluate the degree of dependence. Hereafter, we omit the suffix representing the population. Let $E\left(x_{\alpha}\right)=0$ without loss of generality. At first we calculate the covariance of $y_{j \alpha}$ and $y_{j \beta}$. Sugiura (1976) gave an asymptotic distribution of the $j$-th latent vector $\boldsymbol{h}_{j}=\left(h_{1 j}, \cdots, h_{p j}\right)^{\prime}$ with $h_{j j}>0$ of a Wishart matrix $W_{p}(N-1, \Sigma)$, where $\Sigma=\operatorname{diag}\left(\lambda_{1}, \cdots, \lambda_{p}\right)$ with $\lambda_{1}>\lambda_{2}>\cdots>\lambda_{p}$ $>0$ assuming $\lambda_{j}$ is simple and $n=N-1$ as follows:

$$
\begin{aligned}
& h_{k j}(k \neq j)=-\frac{a_{k j}}{\lambda_{k}-\lambda_{j}}-\frac{a_{j j} a_{k j}}{\left(\lambda_{k}-\lambda_{j}\right)^{2}}+\frac{1}{\lambda_{k}-\lambda_{j}} \sum_{l \neq j} \frac{a_{k l} a_{l j}}{\lambda_{l}-\lambda_{j}} \\
& -\frac{a_{j j}^{2} a_{k j}}{\left(\lambda_{k}-\lambda_{j}\right)^{3}}+\frac{a_{j j}}{\left(\lambda_{k}-\lambda_{j}\right)^{2}} \sum_{l \neq j} \frac{a_{k i} a_{l j}}{\lambda_{i}-\lambda_{j}}+\frac{a_{k j}}{\left(\lambda_{k}-\lambda_{j}\right)^{2}} \sum_{l \neq j} \frac{a_{l j}^{2}}{\left(\lambda_{l}-\lambda_{j}\right)} \\
& +\frac{a_{j j}}{\lambda_{k}-\lambda_{j}} \sum_{l \neq j} \frac{a_{k l} a_{l j}}{\left(\lambda_{l}-\lambda_{j}\right)^{2}}-\frac{1}{\lambda_{k}-\lambda_{j}} \sum_{l, m \neq j} \frac{a_{k l} a_{l m} a_{m j}}{\left(\lambda_{l}-\lambda_{j}\right)\left(\lambda_{m}-\lambda_{j}\right)} \\
& +\frac{1}{2} \frac{a_{k j}}{\lambda_{k}-\lambda_{j}} \sum_{l \neq j} \frac{a_{L j}^{2}}{\left(\lambda_{l}-\lambda_{j}\right)^{2}}+\text { terms of higher order, } \\
& h_{j j}=1-\frac{1}{2} \sum_{l \neq j} \frac{a_{l j}^{2}}{\left(\lambda_{l}-\lambda_{j}\right)^{2}}-\sum_{l \neq j} \frac{a_{j j} a_{l j}^{2}}{\left(\lambda_{l}-\lambda_{j}\right)^{3}}+\sum_{l, m \neq j} \frac{a_{l j} a_{l m} a_{m j}}{\left(\lambda_{l}-\lambda_{j}\right)^{2}\left(\lambda_{m}-\lambda_{j}\right)},
\end{aligned}
$$

where $a_{k l}=\sum_{a} x_{k a} x_{i a} / n-\lambda_{l} \delta_{k l}$. Considering Sugiura's expressions up to order $n^{-1}$ we can rewrite them as follows:

$$
\begin{aligned}
& h_{k j}(k \neq j)=\frac{-3 a_{k j}}{\lambda_{k}-\lambda_{j}}+O\left(n^{-2}\right), \\
& h_{j j}=1+O\left(n^{-2}\right) .
\end{aligned}
$$

Then the covariance of $y_{j \alpha}$ and $y_{j \beta}$ when $\alpha \neq \beta$ is written by the following

$$
\begin{aligned}
E\left(y_{j \alpha} y_{j \beta}\right)= & E\left(\sum_{l=1}^{p} \sum_{m=1}^{p} h_{l j} x_{l a} h_{m j} x_{m \beta}\right)=\sum_{l=1}^{p} \sum_{m=1}^{p} E\left(h_{l j} x_{l \alpha} h_{m j} x_{m \beta}\right) \\
= & \sum_{l \neq j} E\left(\frac{3 a_{l j}}{\lambda_{j}-\lambda_{l}} x_{l a} x_{j \beta}\right)+\sum_{m \neq j} E\left(\frac{3 a_{m j}}{\lambda_{j}-\lambda_{m}} x_{j \alpha} x_{m \beta}\right)+E\left(x_{j \alpha} x_{j \beta}\right)+O\left(n^{-2}\right) \\
= & \sum_{l \neq j} \frac{3}{\lambda_{j}-\lambda_{l}} E\left(\frac{1}{n} \sum_{r} x_{j r} x_{l \gamma} x_{l a} x_{j \beta}\right)+\sum_{m \neq j} \frac{3}{\lambda_{j}-\lambda_{m}} E\left(\frac{1}{n} \sum_{r} x_{j \gamma} x_{m \gamma} x_{j \alpha} x_{m \beta}\right) \\
& +E\left(x_{j \alpha} x_{j \beta}\right)+O\left(n^{-2}\right) .
\end{aligned}
$$

As the $\boldsymbol{x}_{\alpha}$ and $\boldsymbol{x}_{\boldsymbol{\beta}}$ are independent, and all of the odd-order moments of multivariate normal distribution are zero, we can see

$$
E\left(y_{j \alpha} y_{j \beta}\right)=O\left(n^{-2}\right) \text {. }
$$

Further, let the correlation of $y_{j \alpha}$ and $y_{j \beta}, \operatorname{Cor}\left(y_{j \alpha}, y_{j \beta}\right)=\rho_{j}$, which may be very small as shown on the above, and let the variance of $y_{j a}, \operatorname{Var}\left(y_{j a}\right)=\sigma_{j}^{2}$. Then we have

$$
E\left(y_{j \alpha}-\bar{y}_{j}\right)\left(y_{j \beta}-\bar{y}_{j}\right)=-\frac{1}{N}\left(1-\rho_{j}\right) \sigma_{j}^{2} .
$$


This means that the degree of dependence between $y_{j \alpha}-\bar{y}_{j}$ and $y_{j \beta}-\bar{y}_{j}$ is very weak when $N$ is sufficiently large. Therefore, for large $N$ we may ignore the influence of dependence. Under the same assumption, we have

$$
E\left(\frac{1}{N-1} \sum_{\alpha}\left(y_{j a}-\bar{y}_{j}\right)^{2}\right)=E\left(l_{j}\right)=\left(1-\rho_{j}\right) \sigma_{j}^{2} .
$$

As $E\left(l_{j}\right)$ asymptotically equals $\lambda_{j}$, for our testing problem we have to check the applicability of the A-B test for the following two-sample problem:

$$
H_{0}:\left(1-\rho_{j}^{(1)}\right) \sigma_{j}^{(1)^{2}}=\left(1-\rho_{j}^{(2)}\right) \sigma_{j}^{(2)^{2}} .
$$

Let us show the simulation results of the $A-B$ test under the two situations (Case A, Case B):

Case A: The variables $y_{1}^{(1)}, y_{2}^{(1)}, \cdots, y_{N_{1}}^{(1)}$ are normally distributed $N(0,1)$ with the correlation, $\operatorname{Cor}\left(y_{i}^{(1)}, y_{j}^{(1)}\right)=0.05$, and $y_{1}^{(2)}, y_{2}^{(2)}, \cdots, y_{N_{2}}^{(2)}, N(0,1), \operatorname{Cor}\left(y_{i}^{(2)}, y_{j}^{(2)}\right)$ $=0.05$ :

\begin{tabular}{lccc}
\multicolumn{4}{c}{ Simulation result of the A-B test for $H_{0}$} \\
\hline nominal significance levels & .050 & .100 & .200 \\
\hline actual significance levels & .049 & .101 & .202 \\
\hline$\left(N_{1}=N_{2}=100\right.$, replications $\left.=50000\right)$ & &
\end{tabular}

(In the simulation we gave $5 \%, 10 \%$ and $20 \%$ points of $N(0,1)$ as nominal significance levels in each test, and obtained the actual significance levels, namely the proportion of the rejected cases.)

Case B: The variables $y_{1}^{(1)}, y_{2}^{(1)}, \cdots, y_{N_{1}}^{(1)}$ are normally distributed $N(0,5)$ with the correlation, $\operatorname{Cor}\left(y_{i}^{(1)}, y_{j}^{(1)}\right)=0.2$, and $y_{1}^{(2)}, y_{2}^{(2)}, \cdots, y_{N_{2}}^{(2)}, N(0,10), \operatorname{Cor}\left(y_{i}^{(2)}, y_{j}^{(2)}\right)$ $=0.6$ :

Simulation result of the A-B test for $H_{0}$

\begin{tabular}{lrrr}
\hline nominal significance levels & .050 & .100 & .200 \\
\hline actual significance levels & .051 & .103 & .204 \\
\hline \multicolumn{4}{c}{$\left(N_{1}=N_{2}=100\right.$, replications $\left.=50000\right)$}
\end{tabular}

These simulation results suggest that the A-B test may be applicable to test the equality between the expectations of the sample variances of the dependent variables.

\section{Simulation results when covariance matrices are diagonal}

We examine the reliability of our method by simulation under the assumption of the multivariate normal distributions $N_{p}\left(\boldsymbol{\mu}_{\alpha}, \boldsymbol{\Sigma}_{\alpha}\right)$. In this section we consider only the case when the population covariance matrices are diagonal. The simulation was performed under the following situations:

Case 1. $\quad \lambda_{i}^{(1)}=\lambda_{i}^{(2)}, \quad(i=1,2, \cdots, p)$

Case 2. $\quad \lambda_{i}^{(1)}=\lambda_{i}^{(2)}$ and $\lambda_{j}^{(1)} \neq \lambda_{j}^{(2)}, \quad(i \neq j, j=1,2, \cdots, p)$. 
Our testing procedure must be valid for larger samples. So we examined the property for relatively smaller sample sizes; $N_{1}=N_{2}=100$ and $N_{1}=N_{2}=200$. For each case, 200,000 time simulations were performed.

Table 1 to Table 3 show the simulation results for case 1 and $p=3$. In Table 1 , the second and third largest population latent roots were fixed as $\lambda_{2}=$ 2 and $\lambda_{3}=1$, respectively. The largest population latent root was considered for $\lambda_{1}=3, \lambda_{1}=4$ and $\lambda_{1}=6$.

The case of $\lambda_{1}=3$ shows that the testing results for the largest and second largest latent roots are not good for $N_{1}=N_{2}=100$, however, they are better for $N_{1}=N_{2}=200$. The test was a little unconservative under the null hypothesis for both the largest and second largest latent roots. The case of $\lambda_{1}=4$ shows that the results were improved as compared to the case of $\lambda_{1}=3$. Further, the case of $\lambda_{1}=6$ shows that the actual percentage points corresponding to the nominal $5 \%$ level was $4.9 \%$ for both the largest and the second largest in $N_{1}=$ $N_{2}=100$, and $5.0 \%$ for the largest and $4.9 \%$ for the second largest in $N_{1}=N_{2}=$ 200.

From Table 1 we conclude the following:

(Rl) As $\lambda_{1} / \lambda_{2}$ and $\lambda_{2} / \lambda_{3}$ become large, the accuracy of our testing procedure increases.

(R2) In order to get the accuracy which we require, we need a sample size of $N_{1}=N_{2}=100$ or $N_{1}=N_{2}=200$. An adequate sample size depends on the population situation $\lambda_{1} / \lambda_{2}, \lambda_{2} / \lambda_{3}$.

Table 1. Actual significant levels corresponding to the nominal levels by simulation for case 1 , $p=3, \lambda_{2}=2, \lambda_{3}=1$

\begin{tabular}{c|c|ccc|ccc|ccc}
\hline & & $\lambda_{1}=3$ & $\lambda_{2}=2$ & $\lambda_{3}=1$ & $\lambda_{1}=4$ & $\lambda_{2}=2$ & $\lambda_{3}=1$ & $\lambda_{1}=6$ & $\lambda_{2}=2$ & $\lambda_{3}=1$ \\
\hline$N$ & $H_{0}$ & .050 & .100 & .200 & .050 & .100 & .200 & .050 & .100 & .200 \\
\hline \multirow{2}{*}{100} & $\lambda_{1}^{(1)}=\lambda_{(2)}^{(2)}$ & .042 & .088 & .185 & .048 & .097 & .196 & .049 & .101 & .200 \\
& $\lambda_{2}^{(1)}=\lambda_{2}^{(2)}$ & .045 & .094 & .194 & .048 & .097 & .196 & .049 & .098 & .198 \\
& $\lambda_{3}^{(1)}=\lambda_{3}^{(2)}$ & .050 & .100 & .201 & .051 & .102 & .203 & .051 & .102 & .203 \\
\hline \multirow{2}{*}{200} & $\lambda_{1}^{(1)}=\lambda_{1}^{(2)}$ & .046 & .095 & .194 & .049 & .099 & .200 & .050 & .100 & .200 \\
& $\lambda_{2}^{(1)}=\lambda_{2}^{(2)}$ & .048 & .097 & .199 & .050 & .100 & .199 & .049 & .099 & .199 \\
& $\lambda_{3}^{(1)}=\lambda_{3}^{(2)}$ & .050 & .101 & .202 & .051 & .102 & .201 & .052 & .103 & .203 \\
\hline
\end{tabular}

Table 2. Actual significant levels corresponding to the nominal levels by simulation for case 1 , $p=3, \lambda_{2}=3, \lambda_{3}=1$

\begin{tabular}{c|c|ccc|ccc|ccc}
\hline & & $\lambda_{1}=5$ & $\lambda_{2}=3$ & $\lambda_{3}=1$ & $\lambda_{1}=6$ & $\lambda_{2}=3$ & $\lambda_{3}=1$ & $\lambda_{1}=9$ & $\lambda_{2}=3$ & $\lambda_{3}=1$ \\
\hline$N$ & $H_{0}$ & .050 & .100 & .200 & .050 & .100 & .200 & .050 & .100 & .200 \\
\hline \multirow{2}{*}{100} & $\lambda_{1}^{(1)}=\lambda_{(2)}^{(2)}$ & .044 & .093 & .192 & .047 & .097 & .196 & .049 & .101 & .201 \\
& $\lambda_{2}^{(1)}=\lambda_{2}^{(2)}$ & .048 & .098 & .197 & .049 & .100 & .200 & .050 & .101 & .200 \\
& $\lambda_{3}^{(1)}=\lambda_{3}^{(2)}$ & .051 & .102 & .204 & .052 & .104 & .204 & .051 & .103 & .203 \\
\hline \multirow{2}{*}{200} & $\lambda_{1}^{(1)}=\lambda_{1}^{(2)}$ & .048 & .096 & .195 & .049 & .099 & .198 & .050 & .101 & .200 \\
& $\lambda_{2}^{(1)}=\lambda_{2}^{(2)}$ & .050 & .100 & .199 & .050 & .100 & .200 & .050 & .100 & .201 \\
& $\lambda_{3}^{(1)}=\lambda_{3}^{(2)}$ & .051 & .102 & .203 & .051 & .102 & .203 & .051 & .102 & .202 \\
\hline
\end{tabular}


The principal component scores may be approximately independent when the sample size increases. So the sample size mentioned in above (R2) may also be required. The above statements can be confirmed by Table 2 and Table 3 where simulations were performed under other cases for $\lambda_{2} / \lambda_{3}$.

Table 4 to Table 5 present the simulation results in the case of $\Sigma_{1}=\Sigma_{2}$ and $p=5$. The accuracy of the percentage increases when the ratio of population latent roots $\lambda_{1} / \lambda_{2}, \lambda_{2} / \lambda_{3}$ gets large and the sample sizes get larger. These results turned out to be the same as in the case of $p=3$. Thus we reach the following result:

(R3) As the degree of multi-dimensionality increases, our testing procedure ensures a type I error rate as a nominal value.

Table 6 shows the simulation results for the case of $\boldsymbol{\Sigma}_{1} \neq \boldsymbol{\Sigma}_{2}$ and one latent roots being equal, namely the case 2 . Another situation shows the case of $\lambda_{1}^{(1)}$ $=\lambda_{1}^{(2)}, \lambda_{2}^{(1)} \neq \lambda_{2}^{(2)}$ and $\lambda_{3}^{(1)} \neq \lambda_{3}^{(2)}$. It shows that our percentage points for the exact $5 \%$ and $10 \%$ significant points are $4.8 \%$ and $9.9 \%$ when $N_{1}=N_{2}=100$. Another shows the case of $\lambda_{1}^{(1)} \neq \lambda_{1}^{(2)}, \lambda_{2}^{(1)}=\lambda_{2}^{(2)}$ and $\lambda_{3}^{(1)} \neq \lambda_{3}^{(2)}$. Quite a similar percentage $(4.9 \%, 9.9 \%$ and $19.7 \%)$ was obtained for the exact $5 \%, 10 \%$ and $20 \%$ when $N_{1}$ $=N_{2}=100$.

Table 3. Actual significant levels corresponding to the nominal levels by simulation for case 1 , $p=3, \lambda_{2}=5, \lambda_{3}=1$

\begin{tabular}{c|c|ccc|ccc|ccc}
\hline & & $\lambda_{1}=10$ & $\lambda_{2}=5$ & $\lambda_{3}=1$ & $\lambda_{1}=15$ & $\lambda_{2}=5$ & $\lambda_{3}=1$ & $\lambda_{1}=25$ & $\lambda_{2}=5$ & $\lambda_{3}=1$ \\
\hline$N$ & $H_{0}$ & .050 & .100 & .200 & .050 & .100 & .200 & .050 & .100 & .200 \\
\hline \multirow{2}{*}{100} & $\lambda_{1}^{(1)}=\lambda_{1}^{(2)}$ & .048 & .099 & .198 & .049 & .100 & .200 & .050 & .101 & .202 \\
& $\lambda_{2}^{(1)}=\lambda_{2}^{(2)}$ & .050 & .100 & .200 & .050 & .100 & .201 & .051 & .101 & .202 \\
& $\lambda_{3}^{(1)}=\lambda_{3}^{(2)}$ & .052 & .104 & .205 & .052 & .104 & .205 & .052 & .104 & .205 \\
\hline \multirow{2}{*}{200} & $\lambda_{1}^{(1)}=\lambda_{1}^{(2)}$ & .050 & .100 & .199 & .050 & .101 & .202 & .051 & .101 & .199 \\
& $\lambda_{2}^{(1)}=\lambda_{2}^{(2)}$ & .050 & .099 & .199 & .050 & .100 & .201 & .050 & .100 & .200 \\
& $\lambda_{3}^{(1)}=\lambda_{3}^{(2)}$ & .051 & .102 & .203 & .051 & .101 & .202 & .051 & .102 & .202 \\
\hline
\end{tabular}

Table 4. Actual significant levels corresponding to the nominal levels by simulation for case $1, p=5, \lambda_{3}=3, \lambda_{4}=2, \lambda_{5}=1$

\begin{tabular}{|c|c|c|c|c|c|c|c|}
\hline & & $\begin{array}{c}\lambda_{1}=10 \\
\lambda_{4}=2\end{array}$ & $\begin{array}{l}\lambda_{2}=5 \\
\lambda_{5}=1\end{array}$ & $\lambda_{3}=3$ & $\begin{array}{c}\lambda_{1}=20 \\
\lambda_{4}=2\end{array}$ & $\begin{array}{l}\lambda_{2}=5 \\
\lambda_{5}=1\end{array}$ & $\lambda_{3}=3$ \\
\hline$N$ & $H_{0}$ & .050 & .100 & .200 & .050 & .100 & .200 \\
\hline \multirow[t]{5}{*}{100} & $\lambda 1^{(1)}=\lambda^{2)}$ & .047 & .096 & .195 & .050 & .099 & .199 \\
\hline & $\lambda_{2}^{(1)}=\lambda_{2}^{(2)}$ & .043 & .091 & .189 & .044 & .092 & .190 \\
\hline & $\lambda_{3}^{(1)}=\lambda_{3}^{(2)}$ & .040 & .087 & .181 & .042 & .087 & .183 \\
\hline & $\lambda_{4}^{(1)}=\lambda_{4}^{(2)}$ & .046 & .095 & .193 & .045 & .095 & .193 \\
\hline & $\lambda_{5}^{(1)}=\lambda_{5}^{(2)}$ & .052 & .104 & .205 & .052 & .103 & .204 \\
\hline \multirow[t]{5}{*}{200} & $\lambda 1^{(1)}=\lambda \lambda^{(2)}$ & .049 & .099 & .200 & .050 & .100 & .199 \\
\hline & $\lambda_{2}^{(1)}=\lambda_{2}^{(2)}$ & .048 & .097 & .196 & .047 & .095 & .196 \\
\hline & $\lambda_{3}^{(1)}=\lambda_{3}^{(2)}$ & .045 & .093 & .192 & .047 & .095 & .195 \\
\hline & $\lambda^{(1)}=\lambda_{4}^{(2)}$ & .048 & .098 & .199 & .049 & .097 & .195 \\
\hline & $\lambda_{5}^{(1)}=\lambda_{5}^{(2)}$ & .051 & .102 & .203 & .053 & .103 & .204 \\
\hline
\end{tabular}


Table 5. Actual significant levels corresponding to the nominal levels by simulation for case $1, p=5, \lambda_{3}=5, \lambda_{4}=2, \lambda_{5}=1$

\begin{tabular}{c|c|ccc|ccc}
\hline & & $\begin{array}{c}\lambda_{1}=40 \\
\lambda_{4}=2\end{array}$ & $\begin{array}{c}\lambda_{2}=10 \\
\lambda_{5}=1\end{array}$ & $\lambda_{3}=5$ & $\begin{array}{c}\lambda_{1}=40 \\
\lambda_{4}=2\end{array}$ & $\begin{array}{c}\lambda_{2}=20 \\
\lambda_{5}=1\end{array}$ & $\lambda_{3}=5$ \\
\hline$N$ & $H_{0}$ & .050 & .100 & .200 & .050 & .100 & .200 \\
\hline \multirow{6}{*}{100} & $\lambda_{1}^{(1)}=\lambda_{1}^{(2)}$ & .049 & .100 & .200 & .048 & .097 & .196 \\
& $\lambda_{2}^{(1)}=\lambda_{2}^{(2)}$ & .048 & .098 & .197 & .049 & .099 & .199 \\
& $\lambda_{3}^{(1)}=\lambda_{3}^{(2)}$ & .048 & .099 & .199 & .050 & .102 & .202 \\
& $\lambda_{1}^{(1)}=\lambda_{2}^{(2)}$ & .050 & .102 & .202 & .050 & .101 & .201 \\
& $\lambda_{5}^{(1)}=\lambda_{5}^{(2)}$ & .052 & .104 & .205 & .052 & .103 & .205 \\
\hline \multirow{7}{*}{200} & $\lambda_{1}^{(1)}=\lambda_{1}^{(2)}$ & .050 & .101 & .202 & .050 & .099 & .198 \\
& $\lambda_{2}^{(1)}=\lambda_{2}^{(2)}$ & .049 & .100 & .199 & .049 & .099 & .198 \\
& $\lambda_{3}^{(1)}=\lambda_{3}^{(2)}$ & .048 & .099 & .199 & .051 & .100 & .200 \\
& $\lambda_{4}^{(1)}=\lambda_{4}^{(2)}$ & .050 & .100 & .203 & .051 & .100 & .200 \\
& $\lambda_{5}^{(1)}=\lambda_{5}^{(2)}$ & .051 & .103 & .203 & .053 & .103 & .205 \\
\hline
\end{tabular}

Table 6. Actual significant levels corresponding to the nominal levels by simulation for case $2, p=3$

\begin{tabular}{c|c|ccc|ccc}
\hline & & $\lambda_{1}^{(1)}=4$ & $\lambda_{2}^{(1)}=2$ & $\lambda_{3}^{(1)}=1$ & $\lambda_{(1)}^{(1)}=5$ & $\lambda_{2}^{(1)}=3$ & $\lambda_{3}^{(1)}=1$ \\
& & $\lambda_{1}^{(2)}=4$ & $\lambda_{2}^{(2)}=1$ & $\lambda_{3}^{(2)}=0.5$ & $\lambda_{1}^{(2)}=9$ & $\lambda_{2}^{(2)}=3$ & $\lambda_{3}^{(2)}=0.5$ \\
\hline$N$ & $H_{0}$ & .050 & .100 & .200 & .050 & .100 & .200 \\
\hline \multirow{2}{*}{100} & $\lambda_{1}^{(1)}=\lambda_{1}^{(2)}$ & $(.048$ & .099 & $.203)$ & .600 & .716 & .825 \\
& $\lambda_{2}^{(1)}=\lambda_{2}^{(2)}$ & .752 & .843 & .915 & .049 & .099 & $.197)$ \\
& $\lambda_{3}^{(1)}=\lambda_{3}^{(2)}$ & .755 & .843 & .914 & .750 & .839 & .911 \\
\hline
\end{tabular}

These results show that our testing procedure is applicable in data analysis. So we conclude that

(R4) Our testing procedure is reliable in testing hypotheses of case 2.

This result is only valid in the case of $p=3$, but it may be true for a larger $p$ as well.

\section{Simulation results when covariance matrices are not diagonal}

Our testing statistic is invariant to the orthogonal transformation, but for the confirmation of our method we consider the cases when the population covariance matrices are not diagonal. Table 7 shows the simulation results when $N_{1}=N_{2}=100,200, \Sigma_{1}=\Sigma_{2}$ and $\left(\lambda_{1}, \lambda_{2}, \lambda_{3}\right)=(3,2,1),(5,3,1)$ and $(10,5,1)$. The first case in Table 7 is corresponding to one of the results in Table 1, and they are almost equivalent in accuracy. This is true for the other situations in Table 2 and Table 3 . From these results we can see that the validity of our nonparametric procedure depends on the population latent roots whether the covariance matrices are diagonal or not.

\section{Comparison with $F$ test}

The skewness and kurtosis of the $j$-th sample latent root of Wishart matrix 
Table 7. Actual significant levels corresponding to the nominal ones when covariance matrices are not daigonal

\begin{tabular}{|c|c|c|c|c|c|c|c|c|c|c|}
\hline & $\Sigma=\Sigma_{1}=\Sigma_{2}$ & $\left(\begin{array}{l}2.250 \\
0.375 \\
0.217\end{array}\right.$ & $\begin{array}{l}0.375 \\
2.313 \\
0.758\end{array}$ & $\left.\begin{array}{l}0.217 \\
0.758 \\
1.438\end{array}\right)$ & $\left(\begin{array}{l}3.500 \\
0.750 \\
0.433\end{array}\right.$ & $\begin{array}{l}0.750 \\
3.625 \\
1.516\end{array}$ & $\left.\begin{array}{l}0.433 \\
1.516 \\
1.875\end{array}\right)$ & $\left(\begin{array}{l}6.250 \\
1.875 \\
1.083\end{array}\right.$ & $\begin{array}{l}1.875 \\
6.813 \\
3.356\end{array}$ & $\begin{array}{l}1.083 \\
3.356 \\
2.938\end{array}$ \\
\hline$N$ & $H_{0}$ & $\lambda_{1}=3$ & $\lambda_{2}=2$ & $\lambda_{3}=1$ & $\lambda_{1}=5$ & $\lambda_{2}=3$ & $\lambda_{3}=1$ & $\lambda_{1}=10$ & $\lambda_{2}=5$ & $\lambda_{3}=1$ \\
\hline 100 & $\begin{array}{l}\lambda_{1}^{(1)}=\lambda_{1}^{(2)} \\
\lambda_{2}^{(1)}=\lambda_{2}^{(2)} \\
\lambda_{3}^{(1)}=\lambda_{3}^{(2)}\end{array}$ & $\begin{array}{l}.043 \\
.044 \\
.050 \\
\end{array}$ & $\begin{array}{l}.089 \\
.092 \\
.102\end{array}$ & $\begin{array}{l}.185 \\
.190 \\
.203 \\
\end{array}$ & $\begin{array}{l}.044 \\
.048 \\
.052 \\
\end{array}$ & $\begin{array}{l}.093 \\
.097 \\
.103\end{array}$ & $\begin{array}{l}.191 \\
.197 \\
.205\end{array}$ & $\begin{array}{l}.048 \\
.050 \\
.052\end{array}$ & $\begin{array}{l}.097 \\
.101 \\
.104\end{array}$ & $\begin{array}{l}.198 \\
.201 \\
.204\end{array}$ \\
\hline 200 & $\begin{array}{l}\lambda_{1}^{(1)}=\lambda^{(2)} \\
\lambda_{2}^{(1)}=\lambda_{2}^{(2)} \\
\lambda_{3}^{(1)}=\lambda_{3}^{(2)}\end{array}$ & $\begin{array}{l}.047 \\
.049 \\
.051\end{array}$ & $\begin{array}{l}.095 \\
.098 \\
.101\end{array}$ & $\begin{array}{l}.194 \\
.196 \\
.202\end{array}$ & $\begin{array}{l}.048 \\
.049 \\
.051\end{array}$ & $\begin{array}{l}.097 \\
.099 \\
.102\end{array}$ & $\begin{array}{l}.196 \\
.199 \\
.202\end{array}$ & $\begin{array}{l}.049 \\
.050 \\
.051\end{array}$ & $\begin{array}{l}.099 \\
.100 \\
.102\end{array}$ & $\begin{array}{l}.200 \\
.200 \\
.202\end{array}$ \\
\hline
\end{tabular}

Table 8. Actual significant levels corresponding to the nominal ones by $F$ test

\begin{tabular}{c|c|ccc|ccc|ccc}
\hline & & $\lambda_{1}=3$ & $\lambda_{2}=2$ & $\lambda_{3}=1$ & $\lambda_{1}=5$ & $\lambda_{2}=3$ & $\lambda_{3}=1$ & $\lambda_{1}=10$ & $\lambda_{2}=5$ & $\lambda_{3}=1$ \\
\hline$N$ & $H_{0}$ & .050 & .100 & .200 & .050 & .100 & .200 & .050 & .100 & .200 \\
\hline \multirow{2}{*}{100} & $\lambda_{1}^{(1)}=\lambda^{(2)}$ & .038 & .082 & .176 & .043 & .090 & .188 & .048 & .098 & .197 \\
& $\lambda_{2}^{(1)}=\lambda_{2}^{(2)}$ & .042 & .088 & .185 & .047 & .096 & .195 & .051 & .101 & .202 \\
& $\lambda_{3}^{(1)}=\lambda_{3}^{(2)}$ & .052 & .102 & .203 & .053 & .105 & .207 & .055 & .107 & .208 \\
\hline \multirow{2}{*}{200} & $\lambda_{1}^{(1)}=\lambda^{(2)}$ & .046 & .093 & .191 & .047 & .096 & .195 & .049 & .099 & .197 \\
& $\lambda_{2}^{(1)}=\lambda_{2}^{(2)}$ & .047 & .095 & .194 & .051 & .101 & .201 & .050 & .101 & .201 \\
& $\lambda_{3}^{(1)}=\lambda_{3}^{(2)}$ & .052 & .102 & .203 & .052 & .104 & .203 & .052 & .103 & .205 \\
\hline
\end{tabular}

are given as follows (see Sugiura (1990)):

$$
\begin{aligned}
& \text { Skewness }\left(l_{j}\right)=\frac{2 \sqrt{2}}{\sqrt{n}}+O\left(n^{-3 / 2}\right), \\
& \text { Kurtosis }\left(l_{j}\right)=3+\frac{12}{n}+O\left(n^{-2}\right) .
\end{aligned}
$$

We note that the above values do not depend on $j$. These are the same as the case of the chi-square distribution with $n$ degrees of freedom. The distribution of the sample latent root may be approximated by chi-square. We attempt to apply the $F$ test for dispersion to our testing problem. Table 8 shows the simulation results using the $F$ test for the same situations as those in Table 7 . Two methods, our nonparametric procedure and the $F$ test, are compared using Table 7 and Table 8 . Our method is more reliable than the $F$ test in every situation.

\section{Conclusion}

This paper shows that we may apply our testing procedure under the assumption of a multivariate normal distribution when the population latent roots are fairly separate and the sample sizes are large. It may not be under non-normality. It is necessary to develop a new method that is more widely applicable. Further, our method is not of use for the case where principal components are computed from correlation matrices. Further investigation is 
necessary for this case.

\section{Acknowledgement}

The authors would like to thank the referees and the editor for their valuable comments to improve this paper.

\section{REFERENCES}

[1] Anderson, T. W. (1984). An Introduction to Multivariate Statistical Analysis (2nd ed.), John Wiley.

[2] Ansari, A. R. and R. A. Bradley (1960). Rank-sum tests for dispersions, Ann. Math. Statist., 31, 1174-1189.

[ 3 ] Hollander, M. and D. A. Wolfe (1973). Nonparametric Statistical Methods, John Wiley.

[4] Jackson J. E. (1991). A User's Guide to Principal Components, John Wiley.

[5] Seber, G. A. F. (1984). Mulitivariate Observations, John Wiley.

[6] Sugiura, N. (1976). Asymptotic expansions of the distributions of the latent roots and the latent vector of the Wishart and multivariate $F$ matrices, $J$. Multivariate Anal., 6, 500-525.

[7] Sugiura, N. (1990). Graphs of the distributions of bivariate Wishart roots and their cumulants, J. Japan Statist. Soc., 20, 117-136. 